\title{
21. LIGHT OF THE NIGHT-SKY (LUMINESCENCE DU CIEL)
}

PRESIDENT: J. L. Weinberg.

VICE-PRESIDENT: R. Dumont.

ORGANIZING COMMITTEE: A. S. Asaad, H. Elsässer, R. H. Giese, H. Tanabe, Yu. L. Trutse, G. Weill, R. D. Wolstencroft.

\section{INTRODUCTION}

This triennium has been characterized by an unusually large and diverse number of space observations of both the astronomical and geophysical components of the light of the night sky and surprisingly few ground-based observations. In recent years, Commission 21 reports have changed gradually from an emphasis entirely on atmospheric aspects of the light of the night sky to a more general coverage. In this report we continue this change in emphasis by referring only to the astronomical aspects of the light of the night sky.

An interesting historical overview of the light of the night sky is given in a book of the same title by F. E. Roach and J. L. Gordon, D. Reidel Publ. Co., 1973. A number of papers concerning polarimetric aspects of the light of the night sky are included in Planets, Stars, and Nebulae studied with Photopolarimetry (Ed. by T. Gehrels, Tucson, 1974). An extensive, up-to-date review of the zodiacal light has been given by C. Leinert (Space Sci. Rev. 18, 281, 1975). Since a number of reviews have been published during the triennium and since a number of meetings have been held, especially IAU Colloquium No. 31, this report is intended only as a measure of the activity in topics of interest to Commission 21 - not as a comprehensive review or bibliography. Drs Dumont, Elsässer, Giese, and Tanabe provided material for use in this report.

\section{MEETINGS}

IAU Colloquium No. 31, 'Interplanetary Dust and Zodiacal Light', took place in Heidelberg June 10-13, 1975, co-hosted by Max Planck Institutes für Astronomie und Kernphysik. Reports were given on recent space observations of the zodiacal light and particle fluxes, as well as on studies of particle dynamics, comets and meteors. There is now good agreement between the particle number densities derived from in-situ measurements and the observed zodiacal light brightness; the main contribution to the zodiacal light comes from particles micron-sized and larger. Zodiacal light measurements from Pioneer 10 and Helios A indicate a spatial distribution for the dust between $r^{-1}$ and $r^{-1.5}$. While most zodiacal light observers emphasized the temporal constancy of the zodiacal light and F corona, Levasseur and Blamont reported variations which they correlate with meteor streams. There was considerable discussion of the existence and origin of a sub-micron dust component observed to be moving outward through the solar system. Comets were emphasized as the main source of zodiacal dust, although it is not clear whether production rates from comets are sufficient without additional dust sources. Compositional analysis of dust particles collected in the stratosphere was reported; the compositions are similar to Chondritic meteorites.

Other meetings of particular interest to Commission 21:

1. Zodiacal light results are often presented at the annual meetings of the COSPAR Cosmic Dust Panel. Many of the papers are published in Space Research.

2. All aspects of the light of the night sky, with emphasis on the atmospheric emissions, are included in papers and discussions at the annual meetings of the Association of Geomagnetism and Aeronomy (IAGA) of the IUGG. 


\section{GROUND-BASED OBSERVATIONS OF THE ZODIACAL LIGHT}

There are surprisingly few observers or programs continuing to devote efforts to ground-based studies of the zodiacal light. As noted by Dumont (IAU Colloq. 31), careful ground observations are still needed; especially to provide complete sky coverage to within $30^{\circ}$ of the Sun, which space observations have so far been unable to provide.

A better knowledge of the various sources of errors involved in ground-based zodiacal light observations is now possible, and this is very important in deciding whether some moderate fluctuation of brightness or polarization observed from the ground may be regarded as real. Staude (Astron. Astrophys. 39, 325, 1975) carried out detailed calculations of scattering in the Earth's atmosphere, treating the zodiacal light and Milky Way as extended sources. He suggests that part of the discrepancies among some of the available ground-based results (Elsässer and Haug; Weinberg; Dumont) is due to the empirical formulae used by these authors to correct their observations for tropospheric scattering. The new treatment of the problem by Staude is much more accurate and extensive, and although in many cases there are only slight differences with the previous formulae, it is a good opportunity to improve the accuracy of all groundbased results, especially in observations near the horizon and in regions of small polarization. Tables of these calculations are available from the author.

In the introductory paper (Astron. Astrophys. 38, 397, 1975) of a series devoted to the photopolarimetric data obtained at Teide Observatory over a complete solar cycle, Dumont and Sanchez examine the various sources of errors in zodiacal light ground-based studies and attempt to compute the rms errors due to these sources. Their conclusion is that reliable results may be expected over most of the sky (except for polarimetry in the antisolar cap), much care being required to avoid a large dispersion of the results in the dim regions surrounding the ecliptic poles. In the second paper (Astron. Astrophys. 38, 405, 1975) of the series, the same authors present their mean values of the brightness and polarization degree of the zodiacal light along the ecliptic (provisional off-ecliptic results were given in Astron. Astrophys. 22,321, 1973 and in paper 1.1 of $I A U$ Colloq. 31). Applying the inversion formulae introduced by Dumont (Planetary Space Sci. 21, 2149, 1973) to Haleakala data as well as to Tenerife data, Dumont and Sanchez derive the scattering phase functions for an elementary volume of interplanetary space. These phase functions are somewhat similar to the phase functions of asteroids, as determined by Gehrels et al. Although not completely isotropic, the dust grains seem to have a very moderate, if any, forward scattering power, in disagreement with many previously suggested theoretical phase functions, especially those of dielectric materials.

In observations at Haleakala, Wolstencroft and Bandermann (Montrly Notices Roy. Astron. Soc. 163, 229, 1973; Nature 252, 215, 1974) patrolled the antisolar hemisphere. They found short-term variations in the direction and amount of polarization, which they interpret by non-sperical dust grains whose alignment in the interplanetary magnetic field could reflect the vicissitudes of the solar wind. The level of these variations (brightnesses of the order of $1 S_{10}(\mathrm{~V})$ or a few $S_{10}(V)$ for the totally polarized component) appears to make their reality questionable, when compared to the order of magnitude either of the tropospheric terms determined by Divari (Soviet Astron. 12, 503, 1968) and by Staude (Astron. Astrophys. 39, 325, 1975) or of the probable errors estimated by Dumont and Sanchez (Astron. Astrophys. 38, 397, 1975). In a recent note, Sparrow and Weinberg (Astron. Astrophys. 41, 475, 1975) have argued strongly against the reality of these variations.

The 1973-75 contribution of the Pic-du-Midi program, which shares with Haleakala and with Tenerife the important advantage of being perennial, has been oriented towards the seasonal variations of the zodiacal light (and of the atmospheric continuum). Robley (Ann. Geophys. $29,321,1973$ ) finds the zodiacal brightness at the north celestial pole to have a maximum near the spring equinox and a minimum near the autumn equinox. In discussing this variation, ascribed by the author to the inclination of the zodiacal cloud to the ecliptic plane, Robley confirms once again that the symmetry plane of the zodiacal cloud is close to the invariable plane. In another work (Astron. Astrophys. 41, 385, 1975), Robley derives the oblateness of the zodiacal cloud (lower than previously estimated by other authors) together with an estimate 
of the rate of decrease of the space density of dust in the symmetry plane (significantly higher than most previous figures).

Pfleiderer and Classen (Mitt. Astron. Ges. No. 38, 1976) report on zodiacal light observations in and out of the ecliptic (color $B$ of $U B V$ ). They find the zodiacal light bluer than the Sun in regions out of the ecliptic and a minimum brightness 30 to $40^{\circ}$ from the ecliptic pole.

A photographic photometry of the evening zodiacal cone has been performed by Banos and Koutchmy (Icarus 20, 32, 1973) with solar depressions from 15 to $22^{\circ}$. The maximum of brightness appears shifted several degrees north of the ecliptic. The authors claim that twilight phenomena can be ruled out, as well as airglow and vignetting effects, and that the local enhancement might be ascribed to a disturbance of the interplanetary medium such as those observed in coronal studies. A possible correlation with chromospheric activity is pointed out to reinforce that hypothesis, and the mass of the dust cloud advocated by the authors to explain the shift of their isophotes is $10^{15} \mathrm{~g}$. It must be noted that in ground observations, the maximum brightness at any elevation is always shifted away from the ecliptic when the ecliptic is not vertical.

Misconi (Ph.D. dissertation, State Univ. N.Y., 1975) examined the various forces to be expected on zodiacal dust during solar 'storm' conditions. He also analyzed 38 night's observations obtained by Weinberg at Haleakala in 1966, 1967, and 1968 in a search for solar flare effects on zodiacal light. No changes were found in the shape of the main cone at elongations $>23^{\circ}$ to a limit of approximately $20 S_{10}(V)$ and none were found in the level of brightness from night to night to a limit of approximately $100 S_{10}(V)$. In a related result, Munro (IAU Colloq. 31) found less then $5 \%$ variation in the $F$ corona in observations between May 1973 and February 1974 with the Skylab white light coronagraph. Weinberg and Misconi are deriving an observational model of the brightness and polarization of the zodiacal light based on morning and evening observations of the main cone on a number of nights, including observations to high ecliptic latitudes. Observations from Haleakala by Weinberg's group during the triennium were limited to support of observations from Skylab and Pioneer 10 and 11 (including simultaneous multicolor observations of the north celestial pole from Skylab and from Haleakala in June 1973) and to special programs such as detailed mapping of the brightness and polarization in a $40 \times 60^{\circ}$ region of sky containing the Gegenschein.

\section{SPACE OBSERVATIONS OF ZODIACAL LIGHT}

During the report period the zodiacal light was observed from balloons, rockets, manned and unmanned spacecraft, the Pioneer 10/11 deep space probes, and the Helios A close-in solar probe. Observations are planned from a second Helios solar probe, scheduled for launch in January 1976. Many of the results were presented or reviewed at IAU Colloq. 31. They will be mentioned only briefly here.

\section{A. Balloons}

Frey et al. (Astron. Astrophys. 36, 447, 1974) observed at four colors from $3500 \AA$ to $8200 \AA$ with the balloon-borne telescope THISBE. Observations at $5000 \AA$ confirmed the existence of negative polarization at large elongations in the ecliptic. Hofmann et al. (Nature $243,140,1973$ ) also used THISBE, to observe at $2.4 \mu \mathrm{m}$. No deviation from solar color was found in these observations.

\section{B. Rockets}

Sandford et al. (Astrophys. J. 181, L15, 1973) observed the inner zodiacal light in four colors during the July 1972 eclipse. The data were degraded by scattered light, but they suggest a reddening at very small elongations. Leinert et al. (Astron. Astrophys. 30, 411, 1974) made the first observations of the inner zodiacal light (at elongations 15,21 , and $30^{\circ}$ ) during the day and outside of eclipse. The zodiacal light was found to be slightly reddened, and the minimum 
to maximum brightness ratios at each elongation were found to be approximately 0.32 , in agreement with results at $90^{\circ}$ elongation (Sparrow and Ney, Astrophys. J. 174, 705, 1972; Dumont and Sanchez, Astron. Astrophys. 22, 321, 1973).

\section{Manned Spacecraft}

Photographs of the inner zodiacal light were obtained from the dark side of the Moon (shadowed from sunlight and earthshine) from Apollo 15, 16, and 17 (Mercer et al., in Space Res. 13, 1025, 1973; MacQueen et al., Planetary Space Sci. 21, 2173, 1973). During Apollo 17, observations were made of color and polarization, showing the zodiacal light within $15^{\circ}$ of the Sun to be reddened near the ecliptic (Mercer et al., in NASA SP-330, 34-1, 1973). An ultraviolet spectrometer used on Apollo 17 found no excess brightness in the zodiacal light at $1470 \AA$ (Lillie, private commun.). Ten-color observations of the antisolar hemisphere were made with a photoelectric polarimeter during Skylab missions SL-2 and SL-3 (Weinberg, et al., Space Sci. Instr. 1, 138, 1975). The polarized brightness of zodiacal light was found to have the color of the Sun (Sparrow et al, , IAU Colloq. 31).

\section{Unmanned Spacecraft}

OSO-5 observations at $90^{\circ}$ elongation, covering a period of approximately six years, find the zodiacal light to be unchanging to better than $\pm 10 \%$ (Burnett, IAU Colloq. 31). Stray light has limited OSO-6 observations to regions near the antisolar point. Large, short-term changes were observed in the direction and amount of polarization (Roach et al,, in Planets, Stars, and Nebulae, Tucson, 794, 1974) and in the brightness in this region. Roach et al. (Planetary Space Sci. 21, 1185, 1973) suggest that the brightness changes result from scattering by dust near the Earth-Moon system. Short and long period changes were found in D2A observations at $90^{\circ}$ elongation (Levasseur and Blamont, Nature 246, 26, 1973 and $I A U$ Colloq. 31). A 10\% annual variation or asymmetry was found that could be attributed to the invariable plane. Burnett $e t$ al. (Nature 249, 639, 1974) attribute at least part of the short term changes to scattered moonlight. Satellite D2B, launched successfully in the fall of 1975, is making observations of the Gegenschein in bands at 2800,3600 , and $4200 \AA$ (Maucherat and Cruvellier; and Llebaria; IAU Colloq. 31).

\section{E. Space Probes}

Sky mapping observations have been made periodically since the launch of Pioneer 10 (March 1972) and Pioneer 11 (April 1973) with the imaging photopolarimeters IPP's (Weinberg and Hanner) and, at a fixed angle with respect to the spacecraft spin axis, with the asteroidmeteoroid detectors Sisyphus (Soberman and Zook). The Gegenschein was observed by the IPP when the Pioneer 10 spacecraft antisun direction was $3.4^{\circ}$ from the earth antisun direction and when the spacecraft was far from the earth (Weinberg et al. in Space Res. 13, 1187, 1973). IPP observations in the ecliptic at elongations greater than $90^{\circ}$ show no brightness change (i.e., negligible zodiacal light) beyond 3.3 AU (Hanner et al., J. Geophys. Res. 79, 3671, 1974). For a simple power law distribution, IPP and Sisyphus observations both find that the zodiacal light decreases approximately as $r^{-2}$, where $r$ is the heliocentric distance of the spacecraft. Brightness and polarization were measured from Helios $A$ with photometers scanning the sky at ecliptic latitudes $-15,-30$, and $-90^{\circ}$ (Pitz et al., IAU Colloq. 31). Preliminary results indicate a factor of 14 increase in brightness from $1 \mathrm{AU}$ to $0.3 \mathrm{AU}$ (Link et al., IAU Colloq. 31) with the brightness changing approximately as $r^{-1.3}$. There was no indication of a vaporization zone in the closest angular approach $\left(18 R_{\odot}\right)$ of the $15^{\circ}$ photometer. The Helios B photometers will scan the sky at ecliptic latitudes 15,30 , and $90^{\circ}$.

Satellite observations and analysis are continuing with OSO 5 (Burnett et al., Univ. of Minnesota), Pioneer 10/11 (Weinberg and Schuerman, State Univ. N.Y.; Soberman, GE Phila.), and Helios A (Leinert et al., MPI-Astronomie, Heidelberg). Other satellite data still being analyzed: Apollo 15-17 (Mercer, Inst. for Sci. Space Res.; MacQueen et al., High Altitude Observ.; Lillie, 
Univ. of Colo.), Skylab (Weinberg and Sparrow), OAO-2 (Lillie), OSO-6 (F. Roach, J. Roach, B. Carroll, L. Aller), D2A (Levasseur and Blamont, CNRS, Verrièrres-le-Buisson), D2B (Maucherat, Cruvellier, Llebaria; CNRS, Marseille).

\section{PHOTOMETRY OF LUNAR LIBRATION REGIONS}

K. Kordylewski (private commun.) reports that visual observations of $L_{4}$ and $L_{5}$ were conducted in Africa on 9 occasions in 1973 and 6 occasions in 1974. Both clouds were found to have orbital elements differing strongly from the elements of the moon's orbit. From an analysis of OSO-6 observations, J. Roach (Planetary Space Sci. 23, 173, 1975) finds that particles near $L_{4}$ and $L_{5}$ contribute $20 S_{10}(V)$ to the Gegenschein. Three-color observations of backscattering from $L_{4}$ and $L_{5}$ give color indices slightly bluer than the average background (J. Roach, $I A U$ Colloq. 31). Röser (IAU Colloq. 31) has examined the dynamics of particles near $L_{4}$ and $L_{5}$ and finds that only particles with $\rho d \leq 12 \times 10^{-4} \mathrm{~g} \mathrm{~cm}^{-2}(\rho=$ mass density, $d=$ diameter $)$ can contribute to such clouds. Using near-earth fluxes for these particles, he finds a brightness substantially less than the $20 S_{10}(V)$ found by J. Roach. Munro et al. (Planetary Space Sci. 23, 1313, 1975) and Ross (IAU Colloq. 31,) were unable to detect forward scattering from particles in lunar libration regions using the Skylab white light coronagraph. An upper limit to the brightness was determined to be $2.5 \times 10^{-11}$ of the brightness of the mean solar disk.

\section{INTERPLANETARY DUST}

The spatial distribution of interplanetary dust grains close to the ecliptic plane has been found less ambiguous but size dependent by interpretations of recent results from space probes. For larger particles $\left(210^{-9} \mathrm{~g}\right.$ ), Alvarez et al. (COSPAR 1974) found from Pioneer 10 and $11 \mathrm{a}$ general decrease with solar distance $r$ slower than $r^{-1}$ or perhaps even constant (Humes et al., $J$. Geophys. Res. 79, 3677, 1974), no concentration in the asteroid belt, a gap between 1.16 and $1.7 \mathrm{AU}$, and an increase ( 1 to 2 orders of magnitude) near Jupiter. Neste and Soberman (in Space Res. 14, 1974) and Neste (Ph.D. dissertation, Drexel Univ., 1974) found from the Pioneer Sisyphus experiment that the cumulative particle concentration (particles $35 \mu \mathrm{m}$ to $1 \mathrm{~cm}$ ) is relatively constant from 1 to $3.5 \mathrm{AU}$, with some evidence of planetary sweeping effects near Earth and Mars. For zodiacal light (Gegenschein) they report a decrease of intensity $\sim r^{-2}$ out to $2.25 \mathrm{AU}$ and then a more rapid decrease outward. A similar effect was reported by Weinberg et al. (in Space Res. 13, 1187, 1973) and by Hanner and Weinberg (Sky Telesc. 45, 217,1973 ). If the scattering properties of the particles do not change significantly with $r$, then this corresponds to a decrease of particle number density $n$ as $\sim r^{-1}$. For the inner regions $r=0.75$ to $1.09 \mathrm{AU}$, Rhee (Geophys. Res. Letters 1, 345, 1974) obtained $n \sim r^{-1}$ between $0.1 \mathrm{AU}$ and $0.5 \mathrm{AU}$ by evaluation of Pioneer 8 and 9 data and by model computations. For $r>0.5 \mathrm{AU}$, the behavior is different for different size ranges. The space density becomes essentially zero beyond $3.3 \mathrm{AU}$ for small $(<1 \mu \mathrm{m})$ particles but shows no variations larger than about $50 \%$ between 1 and $5 \mathrm{AU}$ for particles $>10^{-9} \mathrm{~g}$. From an analysis of Helios A zodiacal light data, Link et al. ( $I A U$ Colloq. 31) find $n \sim r^{-1.3}$ between 0.08 and 1.5 AU. Using volume scattering functions of ground-based zodiacal light measurements, Dumont and Sanchez (Astron. Astrophys. 35, 405, 1975) found $n(r)$ to go as $r^{-1.2}$, in agreement with Pioneer 10 and Helios A results. Distribution functions close to $r^{-1}$ derived for zodiacal light particles close to the ecliptic suggest that Mie theory is not appropriate, since the increase of the computed Mie scattering functions for small scattering angles gives an increase of $n(r)$ towards the Sun $\left(n \sim r^{-0.1}\right.$ to $\left.r^{-0.5}\right)$ which is too low (Giese, Planetary Space Sci. 21, 513, 1973).

The 3-dimensional distribution of interplanetary dust was discussed by several authors. In their physical and dynamical study of some 20000 radar meteors, Southworth and Sekanina (SAO Spec. Prog. Rept. 1973) derive lines of equal space density, which show a minimum of concentration in the ecliptic at $0.7 \mathrm{AU}$ and a maximum close to $2 \mathrm{AU}$ with a strong concentration of dust toward the ecliptic plane. From the pole-to-ecliptic brightness ratio of the zodiacal light and the run of intensity at $90^{\circ}$ elongation, Giese (Eldo-Cecles/Esro-Cers Sci. Tech. 
Rev. 7, 43, 1975) excluded models with an ellipsoidal density distribution and models of the Gauss type $n \sim r^{-\nu}$, exp $\left(-\gamma z^{2}\right)$, where $z$ is the altitude above the ecliptic plane. Fan models such as $n \sim r^{-\nu} \exp \left(-h \sin \beta_{\odot} \mid\right)$, where $\beta_{\odot}$ is the heliocentric latitude, show the least contradictions to the observational data. A similar result, $n \sim r^{-1} \exp \left(-2.6\left|\sin ^{1.3} \beta_{\odot}\right|\right)$, was derived for $\nu=1$ by Leinert $e$ t al. (Astron. Astrophys. in press) using observations in circles about the Sun at an elongation of approximately $16^{\circ}$. In this work it was also found that the plane of symmetry $\left(i=3.7^{\circ} \pm 0.6^{\circ}\right)$ deviates from the invariable plane towards the planes of the inner planets in the inner regions $r<1 \mathrm{AU}$. Interpreting his Tenerife observations, Dumont ( $I A U$ Colloq. 31) arrives at an ellipsoid model with a 7:1 ratio of axes. Using exponential laws for number density in and out of the ecliptic, Robley (Astron. Astrophys. 41, 385, 1975; Compt. Rend. Acad. Sci. Paris 279, 1974) investigated the flattening of the zodiacal cloud and the inclination $\left(<2^{\circ}\right)$ of the symmetry axis of the zodiacal cloud with respect to the ecliptic. Finally, three models (Gauss, ellipsoid, fan) were used by Fechtig et al. (J. Geophys. Res., in press) to suggest combined zodiacal light and dust detector measurements on an out of ecliptic mission.

Much work was directed to understanding dynamical processes, sources, and sinks of the zodiacal cloud. Mukai et al. (Publ. Astron. Soc. Japan 26, 445, 1974) and Mukai and Mukai (Publ. Astron. Soc. Japan, 25, 481, 1973) investigated orbit stabilization by sublimation counteracting the Poynting-Robertson effect and production of heliocentric concentrations at $4 R_{\odot}$ (graphite-like grains) and $9 R_{\odot}$ (silicate, obsidian) in agreement with $2.2 \mu \mathrm{m}$ observations. The wavelength dependence of the relevant refractive indices (graphite, silicate, ice grains) was taken into account. The same was done by Lamy (Astron. Astrophys. 33, 191, 1974; Astron. Astrophys. 35, 197, 1974; IAU Colloq. 31) who, in a series of publications, investigated the dynamics of interplanetary grains under the aspect of increasing radiation pressure by sublimation (using Mie calculations of efficiency factors and improved formulae for vapor pressure). The dynamics of particles vaporizing near the sun was studied further by Sekanina (IAU Colloq. 31), who refers to rapid elimination of such particles in hyperbolic orbits. Schwehm (IAU Colloq. 31) also discussed light pressure on interplanetary particles with respect to the size- and wavelength-dependence of refractive indices. A possible process to generate fine particles which could be brought into hyperbolic orbits by light pressure is rotational bursting (spinning up due to radiation effects: Radziewski-effect, windwill effect). Paddack (Ph.D. dissertation, Catholic Univ. of America, 1973) and Paddack and Phee (IAU Colloq. 31) estimate this effect as 1 to 2 orders of magnitude faster than the Poynting-Robertson effect in removing particles from the zodiacal cloud, while Sparrow (Geophys. Res. Letters 2, 255, 1975) feels that this is overestimated due to uncertainties in determining fundamental constants (moment-arm). Bandermann (Monthly Notices Roy. Astron. Soc. in press) pointed out that radiation forces on strongly non-spherical particles can mask the Poynting-Robertson effect and even change orbital inclination.

Evidence for hyperbolic cosmic dust particles (Berg and Grun, Space Res. 13, 1973) stimulated discussion of possible sources. Hemenway et al. (Space Res. 13, 1973) and Hemenway (IAU Colloq. 31) argue that such particles ('stardust') are produced in cool regions of the solar atmosphere and that this explains the unexpected high rate of heavy elements found in some of his particle collections. Collisional breakup of meteoroids $\left(10^{-6}\right.$ to $\left.10^{-8} \mathrm{~g}\right)$ and production of hyperbolic orbits ( $\beta$-meteoroids) due to light pressure on the fragments was found to be sufficient to cause the observed Pioneer results (Zook and Berg, Planetary Space Sci. 23, 183, 1975). The density of parent meteoroids causing $\beta$ meteoroids increases with $r$ near $1 \mathrm{AU}$, some anisotropy of microcraters on lunar surface rocks can be expected, and dust production is not a significant source of interstellar dust (Zook, Planetary Space Sci. 23, 1391, 1975).

In the near Earth environment anisotropies of particle fluxes were also observed in space and time. Such fluctuations were related to lunar origin (secondary ejecta), to collisions of particles, and to fragmentation in the outer atmosphere (Bedford, Planetary Space Sci. 23, 1451, 1975; Hoffmann, et al., Planetary Space Sci. 23, 215, 1975, Planetary Space Sci. 23, 985, 1975; Fechtig, IAU Colloq. 31). These effects were also found responsible for the enhancement of near Earth fluxes compared to interplanetary measurements. Recent calculations by Dohnanyi 
(IAU Colloq. 31) confirm the possibility of a lunar origin of some of the fluctuations and the agreement of frontfilm and grid fluxes with quantitative estimates of the flux of hyperbolic meteoroids produced by collision. A special flux enhancement observed by HEOS 2 was identified as due to particles released by Comet Kohoutek near 4 to 5 AU (Hoffmann et al., IAU Colloq. 25, 1975). Levasseur and Blamont (IAU Colloq. 31) find optical evidence for scattering by particles in meteor streams (after eliminating the effects of moonlight in their satellite data). After a thorough study of possible effects and reported events, Misconi (see earlier) finds that variations in zodiacal light due to solar flares could be expected only at small elongations.

As noted earlier, rocket photometry by Nishimura and balloon measurements by Frey et al. show that the color of the zodiacal light is close to solar color. They conclude that this suggests grains which are comparable in size to the longest wavelength employed and that sub micron particles are not compatible with the observed brightnesses. This also agrees with calculations on the color dependence of zodiacal light models (Giese et al., Planetary Space Sci.21, 2061, 1973). There is further evidence that zodiacal light should be produced mainly by particles much larger than $1 \mu \mathrm{m}$. If the cumulative flux curve derived from lunar samples and from HEOS data is converted into a distribution of particle sizes and is used to calculate the expected brightness of zodiacal light in the ecliptic plane, the brightness is in agreement with observations and $80 \%$ is due to particles in the size range $>10 \mu \mathrm{m}$ (Giese and Grün, IAU Colloq. 31; Leinert et al., Astron. Astrophys., in press). The average scattering function of a volume element of interplanetary dust was derived empirically from observations. Dumont (Compt. Rend. Acad. Sci. Paris 278, 537, 1974 and IAU Colloq. 31) and Dumont and Sanchez (Astron. Astrophys. 38, 405, 1975) used the gradient of zodiacal light brightness in the ecliptic to derive the volume scattering function. Leinert et al., Astron. Astrophys., in press) found volume scattering functions by inversion of the integration over the line of sight from zodiacal light measurements at different elongations, adopting a $1 / r$ law for the radial density distribution. It is now necessary to investigate in the laboratory possible types of larger (1 to $100 \mu \mathrm{m})$ grains which might produce such scattering functions. For grains corresponding to the micron size range, microwave analog measurements $(35 \mathrm{GHz})$ have been performed on nonspherical particles of dielectric and absorbing materials (Zerull, IAU Colloq. 31; Zerull and Giese, in Planets, Stars, and Nebulae, Tucson, 901, 1974). A theoretical investigation to interpret zodiacal light polarization and brightness by Fresnel reflection of grains larger than a few $\mu \mathrm{m}$ was performed by Matsumoto (Publ. Astron. Soc. Japan 25, 469, 1973) who finds that refractive indices such as $m=0.3-0.61$ could explain polarization and brightness at medium solar elongations. Large particles, by themselves, do not seem to be able to produce the polarization observed at large elongations.

\section{BACKGROUND STARLIGHT}

In recent years, techniques developed and used from space have made it possible to measure the background galactic radiation in a wide spectral range from soft X-rays to radio waves. However, in this report we will restrict ourselves to the visual, infrared, and ultraviolet. A review of this field, including the history, is given by Roach and Gordon in their book The Light of the Night Sky (D. Reidel Publ. Co., 1973).

An important result was the first direct measurement of the background starlight in the absence of airglow and of zodiacal light. Measurements from Pioneer 10 were made in blue and red bands as part of a program to map the background light, and the first results were reported by Weinberg et al. (J. Geophys. Res. 79, 3665, 1974). Confirmation of Pioneer 10's measurements was carried out by Tanabe and Mori (IAU Colloq. 31), using star counts on the Palomar Sky Survey Atlas. Additional results in this continuing program have been published by Tanabe in Publ. World Data Center C2, Airglow, Tokyo Astronomical Observatory, 1973.

Most of the discrepancy between Elsässer and Haug's measurements of the Milky Way surface brightness and Roach and Megill's calculations based on star counts in Kapteyn's selected areas was removed by Sharov and Lipaeva's (Soviet Astron. 17, 69, 1973) corrections to the photometric $B$ scales of the star catalogs. Miller and MacCarthy (Astron.J. 79, 1294 and 1335, 
1974) presented seven new standard $U B V$ photoelectric sequences for southern Milky Way fields, as a part of the extensive work of B. J. Bok's group. Each sequence, which contains at least 16 stars and has a faint magnitude limit of at least $V=15.3$, would be a good standard for future star counts in the southern sky.

Efforts to determine the state of polarization of the background light have also been made. From polarimetry at $5080 \AA$, Wolstencroft and Bandermann (Monthly Notices Roy. Astron. Soc. $163,229,1973$ ) have found two regions of enhanced linearly polarized intensity with an angular extent of approximately $10^{\circ}$ located at $l \mathrm{II}=0^{\circ}, b \mathrm{II}=-5^{\circ}$ and $l \overline{\mathrm{I}}=25^{\circ}, b \mathrm{II}=18^{\circ}$. They also discussed the Milky Way (and zodiacal light) polarization with new observations made mainly in 1973 and 1974 from Mt. Haleakala (IAU Colloq. 31). Although no firmly established circular polarization was found in the zodiacal light, a small circular polarization was found in the Milky Way (Staude et al., in the Proceedings of IAU Symp. 52, 139, 1973). Wolstencroft concludes (same Proceedings, p. 99) that evidence for polarization of the Milky Way is persuasive but not yet conclusive.

Some of the series of papers 'Wavelength Dependence of Polarization' published by a group at the Lunar and Planetary Laboratory, Arizona (Coyne, Astron. J. 79, 565, 1974; Coyne, Gehrels, and Serkowski, Astron. J. 79, 581, 1974; Gehrels, Astron. J. 79, 590, 1974) investigated rotation of the position angle by the interstellar medium, the wavelength of maximum polarization as a characteristic parameter of interstellar grains, and interstellar polarization from 0.22 to $2.2 \mu \mathrm{m}$, respectively. Their extensive studies of the interstellar polarization are an important contribution to the field of background starlight studies. Martin (Planets, Stars, and Nebulae, Tucson, 926, 1974) has presented theoretical considerations of interstellar circular polarization and has discussed details of how aligned, elongated interstellar grains govern the optical properties of the interstellar medium and cause linear dichroism and birefringence.

Two kinds of maps of the diffuse galactic $\mathrm{H} \alpha$ emission have been published. Reynolds $e t$ al. (Astrophys. J. 185, 869, 1973; Astrophys. J. 192, L53, 1974) constructed a contour map of $\mathrm{H} \alpha$ emission from Fabry-Pérot observations in over 300 directions between $l \mathrm{II}=0^{\circ}$ and $240^{\circ}$ and $b \mathbf{I I}=-50^{\circ}$ to $+30^{\circ}$. Sivan (Astron. Astrophys. Suppl. 16, 163, 1974) carried out a photographic survey of $\mathrm{H} \alpha$ emission in the galactic plane with a new, $60^{\circ}$ field, high luminosity camera using a very narrow interference filter, and presented a mosaic of fourteen wide-field $\mathrm{H} \alpha$ plates covering the entire Milky Way.

From infrared polarimetry in the direction of the galactic center, Maihara et al. (Proc. IAU Symp. 52,191, 1973) found the polarization less than $5 \%$ at $2.2 \mu \mathrm{m}$, and Dyck et al. (Astrophys. $J .188, \mathrm{~L} 103,1974)$ detected $2.4 \pm 0.4 \%$ linear polarization at $11 \mu \mathrm{m}$ with the electric vector approximately perpendicular to the galactic plane. A rocket observation of the diffuse galactic radiation in an 85 to approximately $115 \mu \mathrm{m}$ band at $l \mathrm{II} \sim 163^{\circ}$ and $b \mathrm{II}=5^{\circ}$ to $35^{\circ}$ was carried out by Pipher (IAU Symp. 52, 559, 1973). At low latitudes, the measured background intensity was approximately $9 \times 10^{-11} \mathrm{w} \mathrm{cm}^{-2} \mathrm{sr}^{-1}$; the average number density of grains derived from the thermal emission mechanism is consistent with the optically determined measures.

The average intensity of the interstellar ultraviolet diffuse radiation between $912 \AA$ and $2740 \AA$ was calculated by Gondhalekar and Wilson (Astron. Astrophys. 38, 329, 1975) on the basis of a galactic model. A comparison of the computed flux with wide field observations of the ultraviolet background gives reasonable agreement.

Dodd et al. (Monthly Notices Roy Astron. Soc. 171, 329, 1975) have measured the positions, sizes, shapes, and orientations of images of 3000 faint galaxies in an area of $2 \mathrm{deg}^{2}$ near the south galactic pole on a photograph reaching $B=23$ taken by the UK 48-in. Schmidt telescope in Australia. Although they did not measure the apparent magnitudes, this work provides basic information about the extragalactic light.

In principle, comparison of the measured surface brightness of the background light and the integrated starlight derived from star counts should yield the diffuse galactic light (plus extragalactic light) directly. However, due to faintness of the diffuse galactic light and due to difficulties in obtaining and comparing the background measurements and the star count data, reliable values for the diffuse galactic light are not yet available. New space photometries in 
different colors and star counts in wide regions of the sky should soon remove some of the difficulties.

\section{COLLABORATIVE STUDIES}

The subject of Commission 21 has become increasingly 'international' or regional, and there are a number of collaborative studies. Included among these are:

Bordeaux-Tenerife: Dumont, Sanchez (zodiacal light observations and analysis)

Heidelberg-Albany: Leinert, Weinberg (intercomparison of diffuse source calibration)

Bochum-Heidelberg: Giese, Leinert, others (model calculations, zodiacal light)

Albany-Glasgow: Weinberg, Hanner, Clarke (Pioneer 10/11 data analysis)

Albany-Melbourne: Weinberg, Sparrow (Skylab data analysis)

Tokyo-Albany: $\quad$ Tanabe, Weinberg (background starlight)

Toledo-Boulder: Witt, Lillie (diffuse galactic light)

Bochum-Heidelberg: Giese, Grün, others (comparison of optical and flux data)

Paris-Athens:

Honolulu-Boulder-

Koutchmy, Banos (zodiacal light studies)

Rutgers-UCLA:

Phila.-Houston:

F. Roach, J. Roach, Carroll, Aller (OSO-6 data analysis)

Soberman, Zook (Pioneer-10/11 data analy sis)

J. L. WEINB ERG

President of the Commission 